\title{
Exploring the Epidemic Spreading in a Multilayer Metapopulation Network by considering Individuals' Periodic Travelling
}

\author{
Dun Han $\mathbb{D D}^{1,2}$ Qi Shao, ${ }^{1}$ and Dandan Li $\mathbb{D}^{3}$ \\ ${ }^{1}$ Faculty of Science, Jiangsu University, Zhenjiang, Jiangsu 212013, China \\ ${ }^{2}$ Adaptive Networks and Control Laboratory, Department of Electronic Engineering, \\ Research Center of Smart Networks and Systems, School of Information Science and Engineering, Fudan University, \\ Shanghai 200433, China \\ ${ }^{3}$ School of Management, Jiangsu University, Zhenjiang, Jiangsu 212013, China
}

Correspondence should be addressed to Dun Han; hand@ujs.edu.cn and Dandan Li; li90dan0128@163.com

Received 13 January 2020; Accepted 24 March 2020; Published 21 April 2020

Academic Editor: Toshikazu Kuniya

Copyright (c) 2020 Dun Han et al. This is an open access article distributed under the Creative Commons Attribution License, which permits unrestricted use, distribution, and reproduction in any medium, provided the original work is properly cited.

The convenience of transportation brings the diversity of individuals' travelling modes; in this paper, we present an improved epidemic diffusion model in a multilayer metapopulation network. Firstly, we construct the metapopulation network with different travelling ways, and then, the epidemic spreading threshold is calculated by means of the mean-field method. Taking the periodicity of individuals' travelling into account, we further explore the epidemic diffusion model with individuals' periodic travelling and deduce the epidemic spreading threshold using the Perron-Frobenius theorem. Our results show that if all individuals in each area decide to move, the epidemic threshold can be effectively raised while each individual chooses an unbiased region to arrive. In addition, with the increase of individuals' mobility rate or regional heterogeneous infection coefficient, the fluctuation range of the density of infected becomes larger, while the fluctuation period is almost unchanged. However, the change of individuals' periodic motion could cause the change of the fluctuation period of infected density. We try to provide a new perspective for the research of metapopulation.

\section{Introduction}

The study of epidemic diffusion based on dynamic model has a history of more than 200 years. It can be traced back to 1760 when Bernoulli, a Dutch mathematician and physicist, researched the vaccinated smallpox in order to prolong people's life span [1]. Since the small-world [2] and scale-free networks [3] are presented, many scholars have studied the epidemic spreading using dynamic analysis method based on complex network model [4-9] and obtained a lot of better prediction results for the spread of infectious diseases. The use of mathematical models in the study of epidemic dynamics and their mechanisms and their prevention and control provided valuable insights and contributed to draw up the global picture of epidemics occurrence, patterns, and management [10-13]. However, considering the impact of mathematical models in epidemiology, it is important to build them on realistic assumptions [14-16].
The metapopulation model has been developed in ecological modeling [17], which consists of spatially separated subpopulations of a species [18], and individuals can migrate between subpopulations [19]. A metapopulation model is a useful mathematical framework to understand the dynamics of epidemic spreading in spatially separated subpopulations, corresponding to countries, cities, or local communities, which are connected by pathways for human migration and population mobility [20]. Recently, metapopulation dynamics on networks, which incorporates mobility over the subpopulations, local interaction at the subpopulations, and a complex network structure, has gained great research attention [19, 21-23]. Ni and Weng studied the impact of travel patterns on epidemic dynamics in heterogeneous spatial metapopulation networks. Their results showed that the occurrence probability of global outbreaks is significantly dependent on the characteristic travel distance, the characteristic waiting time, and the 
memory effects of human travel [24]. Wang et al. developed a theoretical framework to investigate the safety-information-driven human mobility with metapopulation epidemic dynamics. Their findings uncovered that the critical threshold depends on mobility in such a way that personal execution of safe moves unexpectedly promotes the global spread of a disease, while unsafe moves counterintuitively cause a locally, relatively small outbreak size [25]. Halvor et al. introduced a generalized reaction-diffusion approach to epidemic spreading in a metapopulation modeled as a complex network. They found that the heterogeneous conditions cause the epidemic threshold to be a nontrivial function of the reaction rates, the network's topology, and the crossover population size that separates "village dynamics" from "city dynamics" [26]. Naoki analyzed the effects of diffusion rates on epidemic spreads in metapopulation networks. The results showed that diffusion can suppress epidemics contrasts with that for diffusive SIS dynamics and its variants when individuals are fixed at nodes on static networks [27].

At present, although there are many studies on the diffusion of infectious diseases in the metapopulation networks, few studies have considered the individuals' travelling patterns [28-31]. Especially, the convenience of transportation brings the diversity of individual travel. For example, when people in the region A decide to travel to the region $\mathrm{B}$, they could choose a train, a car, or a plane. In a result, we could regard different travelling modes as different transportation networks. However, the multilayer system is a very effective tool to analyze this case. In this paper, according to the difference of individuals' travelling modes, we propose an epidemic spreading model on the multilayer metapopulation network and then calculate the epidemic spreading threshold by means of mean field method. The theoretical analysis and numerical simulation results shows that if an individual arrives to other subpopulations without bias, the diffusion of diseases can be effectively suppressed. Moreover, empirical observations in biological and social systems show that individuals' travelling behaviors are regular; for example, the individuals in region $\mathrm{A}$ may periodically travel to region $B$. Inspired by the above fact, we analyze the epidemic diffusion model by considering individuals' periodic movement. The results show that the change of individuals' periodic movement leads to the change of fluctuation cycle of infected density.

The rest of this paper is organized as follows. In Section 2, preliminaries and model formulation for the epidemic spreading in the multilayer metapopulation network is proposed. In Section 3, the multilayer metapopulation network with individuals' periodic movement is explored. In Section 4, simulation examples are given to illustrate the theoretical analysis. The conclusion is given in Section 5.

\section{Epidemic Spreading in the Multilayer Metapopulation Network}

2.1. Preliminaries and Model Formulations. For two realvalued column vectors $u=\left(u_{1}, u_{2}, \ldots, u_{N}\right)^{T}, \quad v=\left(v_{1}\right.$, $\left.v_{2}, \ldots, v_{N}\right)^{T} \in R^{N}$, we say $u \prec v$ if $u_{i} \leq v_{i}$ for all $i \in\{1,2, \ldots, N\}$. For a vector $u=\left(u_{1}, u_{2}, \ldots, u_{N}\right)^{T}$, if $u_{i}>0, i=1,2, \ldots, n$, we note $u>0$. The classical SIS epidemic spreading model includes two types of individuals: the susceptible individual $(S)$ and the infected individual $(I)$. Each susceptible individual becomes infected with probability $\lambda$ when it comes into contact with an infected individual, and the infected nodes are cured and become susceptible again with probability $\beta$. The infection dynamics can be identified by the following set of reaction equations: $S+I \stackrel{\lambda}{\longrightarrow} 2 I, I \stackrel{\beta}{\longrightarrow} S$. In SIR epidemic diffusion model, a class of recovered individuals $R$ is added. The corresponding epidemic diffusion model can be described as $S+I \stackrel{\lambda}{\longrightarrow} 2 I$, $I \stackrel{\beta}{\longrightarrow} R$. For the listed two kinds of epidemic spreading systems, in the mixed homogeneous populations, it is easy to calculate the epidemic spreading threshold: $\lambda_{C}=(\lambda / \beta)$, when $\lambda_{C}=(\lambda / \beta)>1$, infectious diseases will always exist, but when $\lambda_{C}=(\lambda / \beta)>1$, infectious diseases will disappear.

In our model, the total population is divided into $n$ subpopulations, each of which is represented by a node of the metapopulation network. We assume that each node $i$ is initially occupied by $n_{i}$ individuals, so the total number of individuals in the whole system is $N=\sum_{i=1}^{n} n_{i}$. Individuals belonging to node $i$ decide whether or not to move at each time step. Without loss of generality, we assume that individuals belonging to region $i$ move to other regions and return to region $i$ after a time step. The probability of moving is $p$, the probability of remaining is $1-p$. If an individual has $L \geq 0$ travelling modes (walking, car, train, ship, airplane, etc.), each travelling mode could be regarded as a layer of metapopulation network (see Figure 1). For an individual in subpopulation $i$ and deciding to move to region $j$, he will choose a travelling mode $l \in[0, L]$ with probability $P_{i j}^{l}=\left(W_{i j}^{l} / \sum_{l=1}^{L} \sum_{j=1}^{n} W_{i j}^{l}\right)$.

2.2. SIS Epidemic Spreading Model. When all individuals in the metapopulation network have finished their moving, the infected density $\rho_{i}$ belonging to region $i$ (including the primitive inhabitants in region $i$ and those who move to other regions) varies as follows:

$$
\rho_{i}(t+1)=(1-\beta) \rho_{i}(t)+\left(1-\rho_{i}(t)\right) \phi_{i},
$$

where $\beta$ represents recovery rate and the value of $\phi_{i}$ indicates the infected probability who belonging to region $i$, and it is specifically expressed as follows:

$$
\phi_{i}=(1-p) \psi_{i}+p \sum_{l=1}^{L} \sum_{j=1}^{n} P_{i j}^{l} \psi_{j},
$$

where $\psi_{i}$ indicates the probability that the susceptible individuals staying in the region $i$ are infected, specifically as follows:

$$
\psi_{i}=1-\prod_{j=1}^{n}\left(1-\lambda_{j i} \rho_{j}(t)\right)^{n_{j \rightarrow i}},
$$

where $\lambda_{j i}$ denotes the probability that the susceptible individuals belong to region $i$ and infected by the infected individuals of region $j ; n_{j \longrightarrow i}$ denotes the total number of 


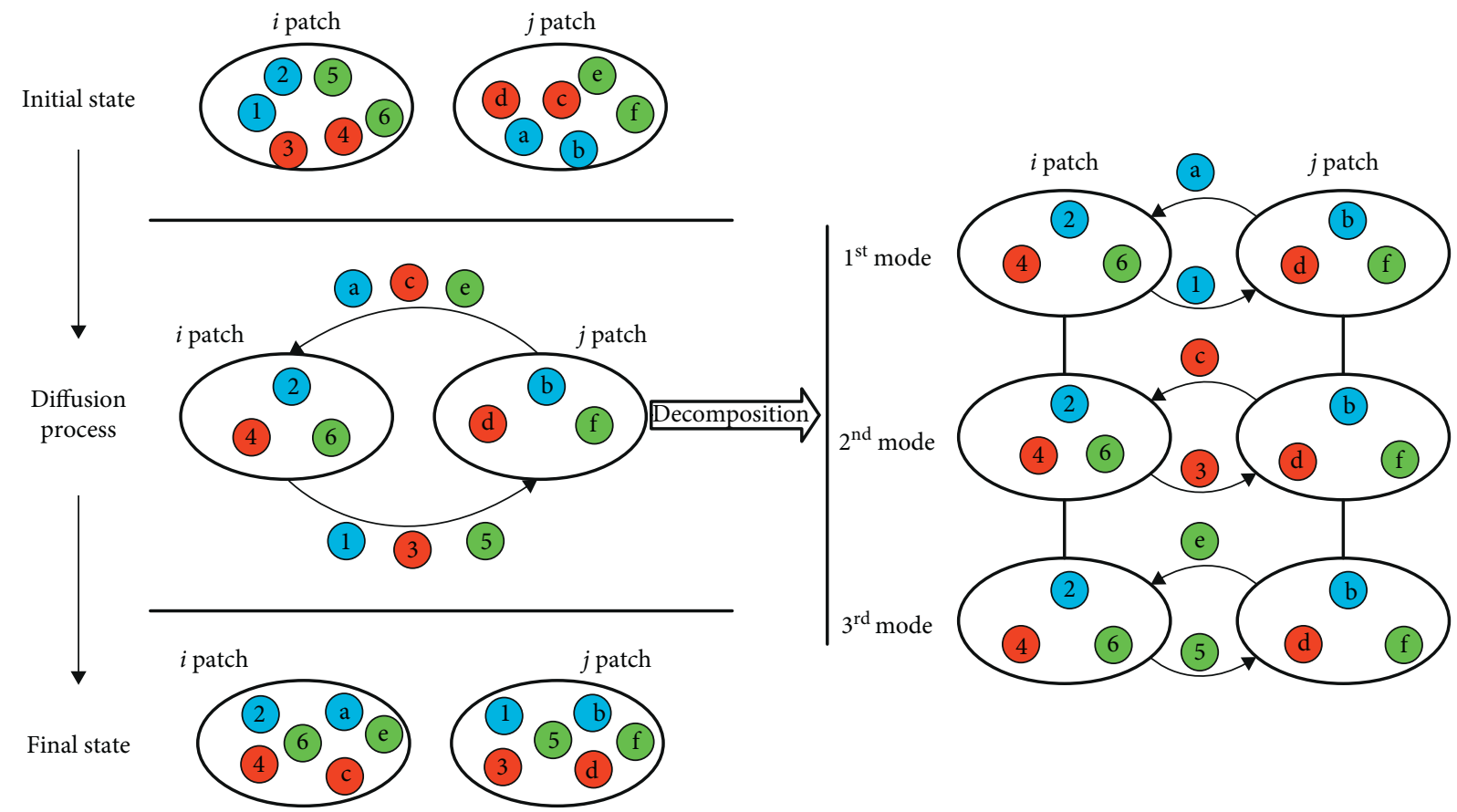

FIGURE 1: Individuals' travelling map with three travelling modes in two subpopulations. Initially, there are six individuals belonging to region $i$ and region $j$, respectively. The individuals with the same color own the same travelling mode.

individuals who move from region $j$ to region $i$, which is described as follows:

$$
n_{j \longrightarrow i}=(1-p) n_{i} \delta_{i j}+p \sum_{l=1}^{L} P_{j i}^{l} n_{j}
$$

where $\delta_{i j}$ represents the Dirac function, if $i=j, \delta_{i j}=1$, and $i \neq j, \delta_{i j}=0$.

We incorporate the comprehensive factors, including the relationship (cooperation, affection, friendship, etc.), the interaction frequency, and the impact of individual environment (for example, one may easy to be in the same room or classroom with the infected one, etc.); therefore, we assume that individuals are easy to be infected by infected individuals in their areas. In this paper, the general expression of infection probability $\lambda_{j i}$ could be presented as follows:

$$
\lambda_{j i}=f\left(\lambda_{0}, \alpha, i, j\right),
$$

where $\lambda_{0}$ represents the underlying infection rate, $\alpha \in[0,1]$ indicates the regional heterogeneous infection coefficient caused by regional differences. The expression of $\lambda_{j i}$ we adopted in this paper is as follows:

$$
\lambda_{j i}=\lambda_{0}\left(1-\alpha\left(1-\delta_{j i}\right)\right) .
$$

Equation (6) shows that $\lambda_{j i} \leq \lambda_{j j}$, i.e., the infected individuals are more likely to infect the susceptible individuals in the same region.

Theorem 1. The epidemic spreading threshold in the metapopulation network considering multiple travelling modes is as follows: $\lambda_{0}^{c}=\left(\beta / \Lambda_{\max }(M)\right)$, where $\Lambda_{\max }(M)$ is the spectral radius of matrix $M, M=\left(M_{i j}\right)_{n \times n}$, where $M_{i k}=[(1-p)$ $\left.n_{i} \delta_{i k}+p \sum_{l=1}^{L} P_{k i}^{l} n_{k}\right](1-p)\left(1-\alpha\left(1-\delta_{k i}\right)\right)+\sum_{l=1}^{L} \sum_{j=1}^{n} P_{i j}^{l} p$ $\left[(1-p) n_{j} \delta_{j k}+p \sum_{l=1}^{L} P_{k j}^{l} n_{k}\right]\left(1-\alpha\left(1-\delta_{k j}\right)\right)$.

Proof. According to equations (1) and (2), we could get that at the equilibrium point:

$$
\beta \rho_{i}^{*}=\left(1-\rho_{i}^{*}\right)\left[(1-p) \psi_{i}+p \sum_{l=1}^{L} \sum_{j=1}^{n} P_{i j}^{l} \psi_{j}\right] .
$$

In order to calculate the epidemic threshold, we linearize equation (7) and neglect the higher order terms of rho $o_{i}^{*}$, we obtain

$$
\beta \rho_{i}^{*}=(1-p) \psi_{i}+p \sum_{l=1}^{L} \sum_{j=1}^{n} P_{i j}^{l} \psi_{j}
$$

Based on equation (3), we can get

$$
\psi_{i}=1-\prod_{j=1}^{n}\left(1-\lambda_{j i} \rho_{j}^{*}\right)^{n_{j \rightarrow i}}=\sum_{j=1}^{n} n_{j \longrightarrow i} \lambda_{j i} \rho_{j}^{*} .
$$

Combining equations (4), (6), (8), and (9), we obtain

$$
\begin{aligned}
\frac{\beta}{\lambda} \rho_{i}^{*}= & \sum_{k=1}^{n}\left[\left[(1-p) n_{i} \delta_{i k}+p \sum_{l=1}^{L} P_{k i}^{l} n_{k}\right](1-p)\left(1-\alpha\left(1-\delta_{k i}\right)\right)\right. \\
& \left.+\sum_{l=1}^{L} \sum_{j=1}^{n} P_{i j}^{l} p\left[(1-p) n_{j} \delta_{j k}+p \sum_{l=1}^{L} P_{k j}^{l} n_{k}\right]\left(1-\alpha\left(1-\delta_{k j}\right)\right)\right] \rho_{k}^{*} .
\end{aligned}
$$

Let $\rho^{*}=\left[\rho_{1}^{*}, \rho_{2}^{*}, \ldots, \rho_{n}^{*}\right]^{T}, M=\left(M_{i j}\right)_{n \times n}$, where 


$$
\begin{aligned}
M_{i k}= & {\left[(1-p) n_{i} \delta_{i k}+p \sum_{l=1}^{L} P_{k i}^{l} n_{k}\right](1-p)\left(1-\alpha\left(1-\delta_{k i}\right)\right) } \\
& +\sum_{l=1}^{L} \sum_{j=1}^{n} P_{i j}^{l} p\left[(1-p) n_{j} \delta_{j k}+p \sum_{l=1}^{L} P_{k j}^{l} n_{k}\right]\left(1-\alpha\left(1-\delta_{k j}\right)\right) .
\end{aligned}
$$

Further, we can get

$$
\frac{\beta}{\lambda_{0}} \rho^{*}=M \rho^{*}
$$

So we can calculate the epidemic spreading threshold as follows:

$$
\lambda_{0}^{c}=\frac{\beta}{\Lambda_{\max }(M)}
$$

Corollary 1. If $p=0$, that is, all individuals stay in their respective subpopulation without moving, we get if $(\lambda / \beta)<\left(1 / \max \left\{n_{i}\right\}\right),(i=1,2, \ldots, n)$, infectious diseases do not break out. However, once $(\lambda / \beta)<\left(1 / \max \left\{n_{i}\right\}\right)$, infectious diseases will break out.

Proof. Let $x=\left[x_{1}, x_{2}, \ldots, x_{n}\right], y=\left[y_{1}, y_{2}, \ldots, y_{n}\right]$, constructing the Lagrangian Functions $L\left(x, y, \lambda_{1}, \lambda_{2}\right)=$ $\sum_{i=1}^{n} x_{i} y_{i}+\lambda_{1}\left(\sum_{i=1}^{n} x_{i}-1\right)+\lambda_{2}\left(\sum_{i=1}^{n} y_{i}-1\right)$, let $\left(\partial L / \partial x_{i}\right)=0$, $\left(\partial L / \partial y_{i}\right)=0,\left(\partial L / \partial \lambda_{1}\right)=0,\left(\partial L / \partial \lambda_{2}\right)=0,1 \leq i \leq n$, we can get $x_{i}=y_{i}=(1 / n),(i=1,2, \ldots, n)$.

Corollary 2. If $p=1$ and the number of individuals in each region is the same at the initial time, i.e., $n_{i} \equiv n_{c},(i=1,2, \ldots, n)$, the epidemic spreading threshold is $\lambda_{0}^{c}=\left(\beta / n_{c}(1-a)\right)$.

Proof. According to equation (11), we can get

$$
M_{i k}=\sum_{l=1}^{L} \sum_{j=1}^{n} P_{i j}^{l} \sum_{l=1}^{L} P_{k j}^{l} n_{k}\left(1-\alpha\left(1-\delta_{k j}\right)\right) .
$$

Let $P_{i j}=\sum_{l} P_{i j}^{l}$, so we can get

$$
\begin{aligned}
M_{i k} & =\sum_{l=1}^{L} \sum_{j=1}^{n} P_{i j}^{l}\left(1-\alpha\left(1-\delta_{k j}\right)\right) n_{k} P_{k j} \\
& =\sum_{j=1}^{n} P_{i j} P_{k j} n_{k}\left(1-\alpha\left(1-\delta_{k j}\right)\right) .
\end{aligned}
$$

If the number of individuals at the beginning of each region is the same, then we obtain

$$
M_{i k}=n_{c} \sum_{j=1}^{n} P_{i j} P_{k j}\left(1-\alpha\left(1-\delta_{k j}\right)\right) \text {. }
$$

Further, we can get

$$
M_{i k}=n_{c}(1-\alpha) \sum_{j=1}^{n} P_{i j} P_{k j}+\alpha P_{i k} P_{k k}
$$

As $\sum_{j} P_{i j}=1$, according to Lemma 1 , we can get

$$
\sum_{k=1}^{n} M_{i k} \geq \sum_{k=1}^{n} n_{c}(1-\alpha) \sum_{j=1}^{n} P_{i j} P_{k j} \geq n_{c}(1-a), \quad i=1,2, \ldots, n .
$$

If and only if $P_{i j}=(1 / n),(1 \leq i, j \leq n)$, the second equal sign was established. Because $\Lambda_{\max }(M) \geq \min \left\{\sum_{j=1}^{n} M_{i j}\right\}$, $(1 \leq i \leq n)$; therefore, the transmission threshold of infectious diseases is $\lambda_{0}^{c}=\left(\beta / n_{c}(1-a)\right)$.

It can be concluded from Corollary 2 that in a multilayer metapopulation network, if $p=1$ and when the individuals move to other regions with equal probability, the epidemic spreading threshold can be effectively increased, i.e., if each individual chooses an unbiased region to arrive, the diffusion of infectious diseases can be effectively suppressed.

Remark 1. In this part, for the convenience of analysis, we replace $\sum_{l=1}^{L} P_{i j}^{l}$ by $P_{i j},(1 \leq i, j \leq n)$. However, in the actual situation, we need to consider different travelling modes and set different value of $P_{i j},(1 \leq i, j \leq n)$.

2.3. SIR Epidemic Spreading Model. In the SIR epidemic spreading model, a new state $R$ is included, so that we can make corresponding improvements to equation (1):

$$
\left\{\begin{array}{l}
\rho_{i}(t+1)=(1-\beta) \rho_{i}(t)+\left(1-\rho_{i}(t)-r_{i}(t)\right) \phi_{i} \\
r_{i}(t+1)=r_{i}(t)+\beta \rho_{i}(t)
\end{array}\right.
$$

where $r_{i}(t)$ is the density of recovered individuals in region $i$ at the time $t$. In order to calculate the epidemic spreading threshold, we linearize equation (19) and neglect the high order of $\rho_{i}^{*}$ and $r_{i}^{*}$, then we obtain

$$
\beta \rho_{i}^{*}=\phi_{i} \text {. }
$$

According to the threshold calculation method in SIS model, we can get a similar result; that is, the epidemic spreading threshold is $\lambda_{0}^{c}=\left(\beta / \Lambda_{\max }(M)\right)$.

\section{Epidemic Spreading in Multilayer Metapopulation Network with Individuals' Periodic Travelling}

In Section 2, we analyze the epidemic spreading model in a multilayer metapopulation network; we next expand the model by considering individuals' periodic travelling. In general, individual travelling is periodic, i.e., individuals may arrive at other regions regularly. For simplicity, we assume that all the individuals have the same travelling period. Therefore, the following changes need be modified to equations (1)-(4):

$$
\rho_{i}(t+1)=(1-\beta) \rho_{i}(t)+\left(1-\rho_{i}(t)\right) \phi_{i}^{*},
$$

where $\phi_{i}^{*}=\left(1-p^{*}\right) \psi_{i}+p^{*} \sum_{l=1}^{L} \sum_{j=1}^{n} P_{i j}^{l} \psi_{j}, \quad \psi_{i}=1-\prod_{j=1}^{n}$ $\left(1-\lambda_{j i} \rho_{j}(t)\right)^{n_{j \longrightarrow i}}, \quad n_{j \longrightarrow i}=\left(1-p^{*}\right) n_{i} \delta_{i j}+p^{*} \sum_{l=1}^{L} P_{j i}^{l} n_{j}$, $p^{*}=p H\left(\sin \pi t / t_{s}\right) . H\left({ }^{*}\right)$ denotes the Heaviside function, and $H(x)=\left\{\begin{array}{ll}1, & x>0 \\ 0, & x \leq 0\end{array}, t_{s}\right.$ is the half period of interpopulation communication. So when the half period $t_{s} \longrightarrow+\infty, 0<t<t_{s}, p^{*}=p$, at this point, there will always be interindividual flow between subpopulations. However, if 
$t_{s}=1$, and $t \in N^{+}$, there will be no inter-individual flow between subpopulations.

Theorem 2 (See Perron-Frobenius theorem [32]). If $M=$ $\left(M_{i j}\right)_{n \times n}$ is an $n \times n$ nonnegative and irreducible matrix, then one of its eigenvalues is positive and greater than or equal to (in absolute value) all other eigenvalues. Such an eigenvalue is called the "dominant eigenvalue" or Perron-Frobenius eigenvalue of the matrix; there is a positive eigenvector corresponding to that eigenvalue; and $\rho(M)$ is equal to the dominant eigenvalue of the matrix.

Lemma 1. The matrix of strong connection graph satisfies Perron-Frobenius theorem. That is, for a strongly connected graph matrix $M$, there is and only one eigenvalue $\lambda=\rho(M)>0$. The corresponding eigenvector $u>0$.
Theorem 3. For system (21), the epidemic spreading threshold is $\lambda_{0}^{c}=\left(\beta / \Lambda_{\max }(\tilde{M})\right)$, where $\Lambda_{\max }(\tilde{M})=$ $\arg _{t} \max \{\rho(\tilde{M})\}, \rho\left(^{*}\right)$ represents the spectral radius of a matrix, $\bar{M}=\left(\widetilde{M}_{i j}\right)_{n \times n}, \widetilde{M}_{i j}=\left(1-p H\left(\sin \pi t / t_{s}\right)\right)(1-\alpha(1-$ $\left.\left.\delta_{k i}\right)\right) n_{k \longrightarrow i}+p H\left(\sin \pi t / t_{s}\right) \sum_{j=} 1^{n} P_{i j}\left(1-\alpha\left(1-\delta_{k i}\right)\right) n_{k \longrightarrow i}$.

Proof. According to equation (21), it can be obtained that at the disease-free equilibrium point:

$$
\begin{aligned}
\frac{\beta \rho_{i}}{1-\rho_{i}}= & \left(1-p H\left(\sin \pi t / t_{s}\right)\right) \sum_{j=1}^{n} \lambda_{0}\left(1-\alpha\left(1-\delta_{j i}\right)\right) n_{j \longrightarrow i} \rho_{j}(t) \\
& +p H\left(\sin \pi t / t_{s}\right) \sum_{l=1}^{L} \sum_{j=1}^{n} P_{i j}^{l} \sum_{k=1}^{n} \lambda_{0}\left(1-\alpha\left(1-\delta_{k i}\right)\right) n_{k \longrightarrow i} \rho_{k}(t) .
\end{aligned}
$$

By deriving $\lambda_{0}$ at both ends of equation (22), one can obtain

$$
\begin{aligned}
\frac{\left(\mathrm{d} \rho_{i} / \mathrm{d} \lambda_{0}\right)\left(1-\rho_{i}\right)-\rho_{i}\left(\mathrm{~d} \rho_{i} / \mathrm{d} \lambda_{0}\right)}{\left(1-\rho_{i}\right)^{2}} & =\left(1-p H\left(\frac{\sin \pi t}{t_{s}}\right)\right) \sum_{j=1}^{n}\left(1-\alpha\left(1-\delta_{j i}\right)\right) n_{j \rightarrow i} \rho_{j}(t)+\lambda_{0}\left(1-\alpha\left(1-\delta_{j i}\right) n_{j \longrightarrow i} \frac{\mathrm{d} \rho_{j}}{\mathrm{~d} \lambda_{0}}\right. \\
& =p H\left(\frac{\sin \pi t}{t_{s}}\right) \sum_{l=1}^{L} \sum_{j=1}^{n} P_{i j}^{l} \sum_{k=1}^{n}\left(1-\alpha\left(1-\delta_{k i}\right)\right) n_{k \longrightarrow i} \rho_{k}(t)+\lambda_{0}\left(1-\alpha\left(1-\delta_{k i}\right) n_{k \rightarrow i} \frac{\mathrm{d} \rho_{k}}{\mathrm{~d} \lambda_{0}}\right.
\end{aligned}
$$

Because at the point of epidemic spreading threshold $\lambda_{0}^{c}$, there must be $\left.\rho_{i}\right|_{\lambda_{0}=\lambda_{0}^{c}}=0,\left(\partial \rho_{i} / \partial \lambda_{0}^{c}\right)>0$, so when $\lambda_{0}=\lambda_{0}^{c}$, we can simplify equation (23):

$$
\begin{aligned}
\beta \frac{\mathrm{d} \rho_{i}}{\mathrm{~d} \lambda_{0}}= & \left(1-p H\left(\frac{\sin \pi t}{t_{s}}\right)\right) \sum_{j=1}^{n} \lambda_{0}\left(1-\alpha\left(1-\delta_{j i}\right)\right) n_{j \longrightarrow i} \frac{d \rho_{j}}{d \lambda_{0}} \\
& +p H\left(\frac{\sin \pi t}{t_{s}}\right) \sum_{l=1}^{L} \sum_{j=1}^{n} P_{i j}^{l} \sum_{k=1}^{n} \lambda_{0}\left(1-\alpha\left(1-\delta_{k i}\right)\right) n_{k \longrightarrow i} \frac{\mathrm{d} \rho_{k}}{\mathrm{~d} \lambda_{0}} .
\end{aligned}
$$

Let $\left(\mathrm{d} \rho_{i} / \mathrm{d} \lambda_{0}\right)=\xi_{i}, P_{i j}=\sum_{l} P_{i j}^{l}$. According to simplification of equation (24), we obtain

$$
\begin{aligned}
\xi_{i}= & \left(1-p H\left(\frac{\sin \pi t}{t_{s}}\right)\right) \sum_{k=1}^{n} \frac{\lambda_{0}}{\beta}\left(1-\alpha\left(1-\delta_{k i}\right)\right) n_{k \longrightarrow i} \xi_{k} \\
& +p H\left(\frac{\sin \pi t}{t_{s}}\right) \sum_{k=1}^{n} \sum_{j=1}^{n} \frac{\lambda_{0}}{\beta} P_{i j}\left(1-\alpha\left(1-\delta_{k i}\right)\right) n_{k \longrightarrow i} \xi_{k} .
\end{aligned}
$$

Let $\tilde{M}=\left(\widetilde{M}_{i j}\right)_{n \times n}, \quad \widetilde{M}_{i j}=\left(1-p H\left(\sin \pi t / t_{s}\right)(1-\alpha(1-\right.$ $\left.\left.\delta_{k i}\right)\right) n_{k \rightarrow i}+p H\left(\sin \pi t / t_{s}\right) \sum_{j=1}^{n} P_{i j}\left(1-\alpha\left(1-\delta_{k i}\right)\right) \quad n_{k \rightarrow i}$. According to equation (25), we can obtain

$$
\xi_{i}=\frac{\lambda_{0}}{\beta} \sum_{k} \tilde{M}_{i k} \xi_{k}
$$

Because $\widetilde{M}_{i k}>0$, and the multilayer metapopulation network is strongly interconnected, let $\Lambda_{\max }(\widetilde{M})=$ $\arg _{t} \max \rho(\tilde{M}), \rho\left(^{*}\right)$ represents the spectral radius of the matrix. According to Theorem 2 and Corollary 2, the epidemic spreading threshold is as follows:

$$
\lambda_{0}^{c}=\frac{\beta}{\Lambda_{\max }(\tilde{M})} \text {. }
$$

Corollary 3. When the half period $t_{s} \longrightarrow+\infty$, i.e., there are always interindividual flows between subpopulations, the epidemic spreading threshold is $\lambda_{0}^{c}=\left(\beta / \Lambda_{\max }(M)\right)$.

Proof. Because when $t_{s} \longrightarrow+\infty$, equation (21) can be equivalent to equation (1), so it can be proved.

\section{Numerical Simulations}

Let $f^{t}=(1 / n) \sum_{i=1}^{n} \rho_{i}(t) n_{i}$ denote the infected density at time $t$, and $f^{\infty}=(1 / n) \sum_{i=1}^{n} \rho_{i}(\infty) n_{i}$ denote the final infected density. We take $n=10$ regions. The number of people in each region follows a uniform distribution, i.e., $n_{i} \sim U(100,500), i=1,2, \ldots, n$, each individual has $L=3$ travelling modes. At each time, the individual randomly chooses one mode to travel. The probability of individuals in region $i$ move to neighbouring region $j$ by means of trips $l=1,2,3$ is $P_{i j}^{l} \sim U(0,1)$ and $\sum_{j} \sum_{l} P_{i j}^{l}=1$. In order to eliminate the effect of randomness on the results, the following experimental results are averaged over 100 times.

Figure 2 depicts the change of infected density $f^{t}$ in the whole metapopulation network over time. It can be seen from Figure 2 that when $\lambda_{0}$ is larger enough, the value of $f^{t}$ increases gradually. However, while the infection rate $\lambda_{0}$ is 


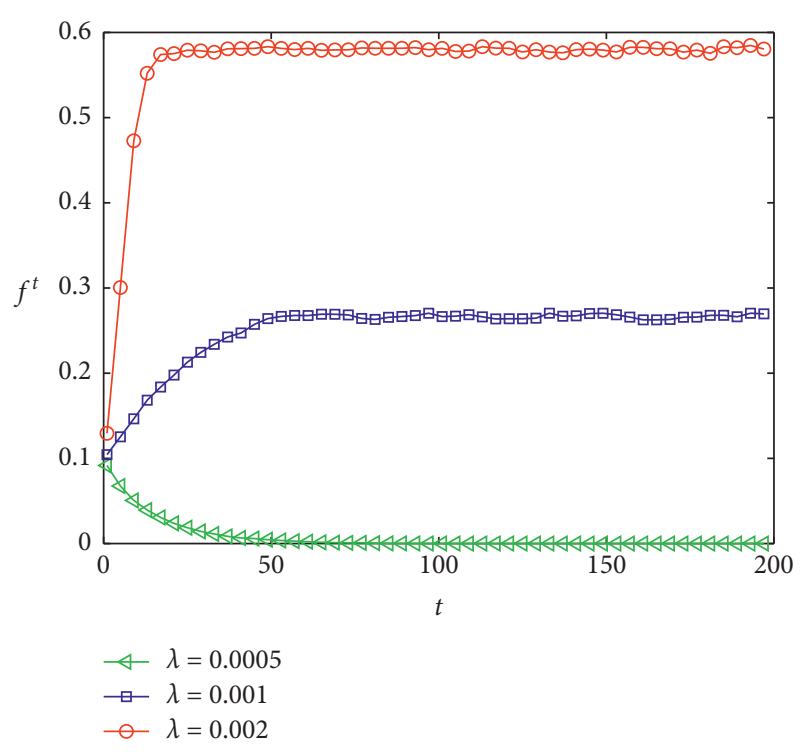

Figure 2: The infected density $f^{t}$ changes with time $t$. With the increase of the infection rate $\lambda_{0}$, the value of $f^{t}$ gradually increases. Parameters are set to be $p=0.5, \beta=0.2, \alpha=0.1$.

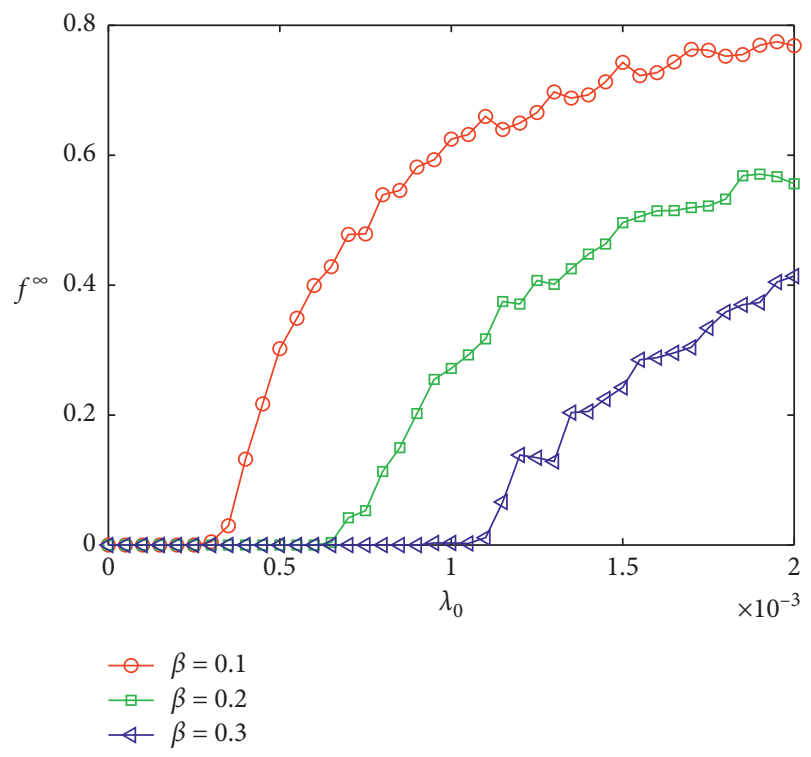

Figure 3: The final infected density $f^{\infty}$ changes with $\lambda_{0}$. With the increase of $\lambda_{0}$, the final infected density $f^{\infty}$ increases gradually. Parameters are set to be $p=0.5, \alpha=0.1$.

smaller, the value of $f^{t}$ decreases gradually until no infected individual exists. Besides, although the interindividual flow exists in the metapopulation network, the value of $f^{t}$ does not show periodic oscillation.

We adopt a small infection rate $\lambda_{0}$. On one hand, the number of individuals in the whole metapopulation is huge; on the other hand, the contact between individuals is frequent. So even at such a small infection rate, there will still be a lot of infected individuals. Figure 3 depicts how the infected density changes with the infection rate $\lambda_{0}$. The

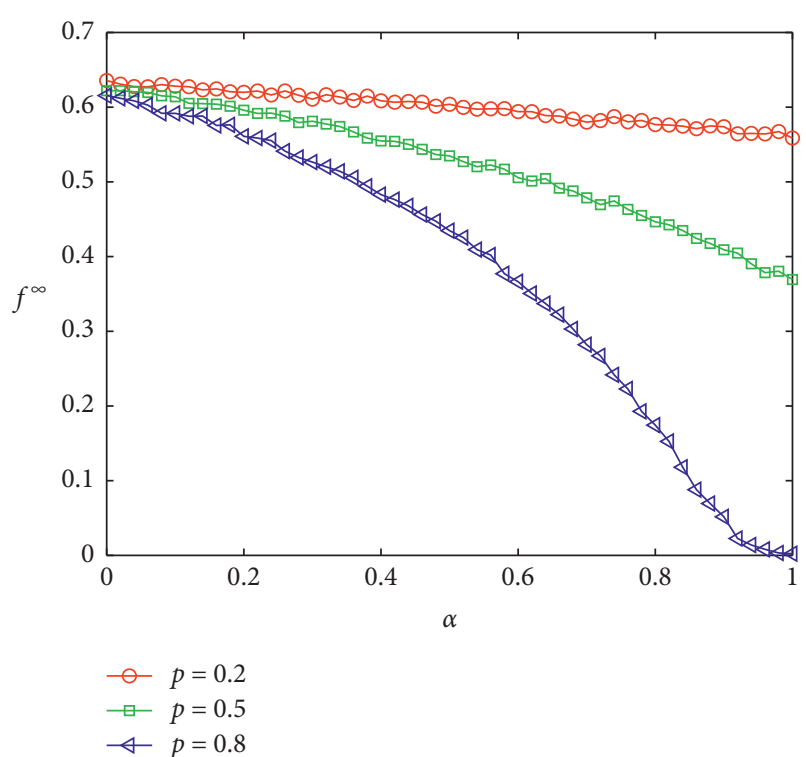

FIgURE 4: The final infected density $f^{\infty}$ changes with the regional heterogeneous infection coefficient. With the increase of $\alpha$, the final density of infected $f^{\infty}$ in the whole system decreases gradually. Parameters are set to be $\beta=0.1, \lambda=0.001$.

theoretical epidemic spreading threshold $\lambda_{0}^{c}=\left(\beta / \Lambda_{\max }(M)\right)$ can be calculated by equation (13). With the increase of infection rate $\lambda_{0}$, the density of infected individuals in the whole system increases gradually, while with the increase of recovery rate $\beta$, the density of infected persons in the whole system decreases gradually. In addition, based on the theoretical epidemic spreading threshold $\lambda_{0}^{c}=\left(\beta / \Lambda_{\max }(M)\right)$, it can be concluded that for the same $\Lambda_{\max }(M)$, the ratio of epidemic spreading threshold under different parameters is $\left(\lambda_{0}^{c 1} / \lambda_{0}^{c 2}\right)=\left(\beta^{1} / \beta^{2}\right)$. This conclusion is proved by the numerical simulation results in Figure 3.

Figure 4 depicts how the final infected density $f^{\infty}$ changes with the regional heterogeneous infection coefficient. One can observe from Figure 4 that the density of the infected individuals decreases gradually with the increase of and $p$. If the probability of infection in the original residence is greater than that in other regions, i.e., $\lambda_{i j} \leq \lambda_{i i},(1 \leq i, j \leq n)$, moving to other regions could effectively reduce the infected risk.

Figure 5 presents the evolution of the infected density $f^{t}$ over time under different half period $t_{s}$. According to Figure 5, we can see that in the multilayer metapopulation network with individuals' periodic travelling, the infected density $f^{t}$ also changes periodically with time. In addition, the larger the individuals' travelling period $2 t_{s}$, the greater the fluctuation period of the density $f^{t}$. This interesting result reveals the diffusion of recurrent infectious diseases to a certain extent.

Figure 6 depicts the evolution of the infected density $f^{t}$ over time at different mobility rates $p$. By comparing Figures $6(\mathrm{a})$ and $6(\mathrm{~b})$, we find the fluctuation range of the infected density increase with $p$ and $t_{s}$, whereas even if the 


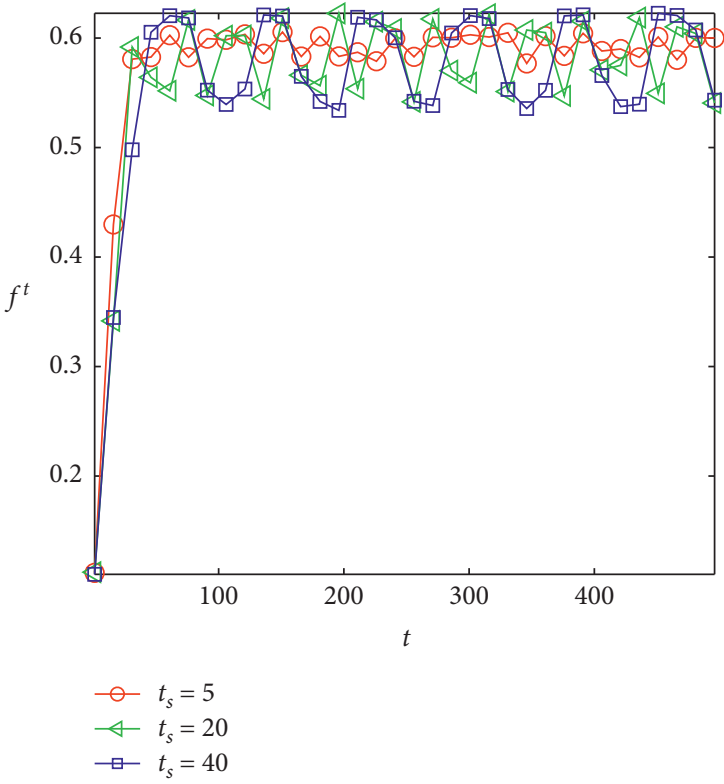

(a)

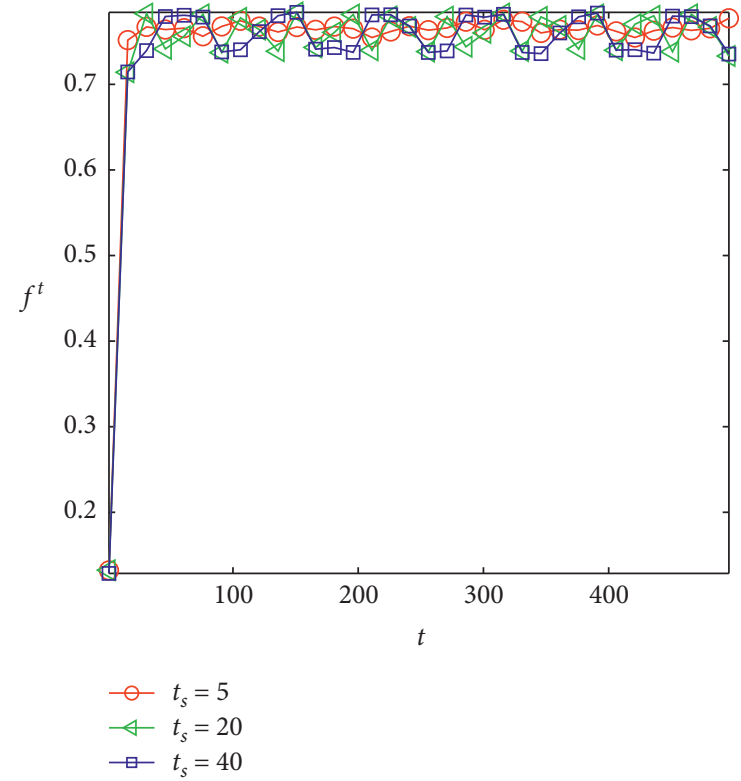

(b)

Figure 5: The evolution of infected density $f^{t}$ over time under different half period $t_{s}$. The parameters are set as follows: $p=0.5, \alpha=0.5$, $\beta=0.1$. (a) The infection rate $\lambda_{0}=0.001$; (b) the infection rate $\lambda_{0}=0.002$.

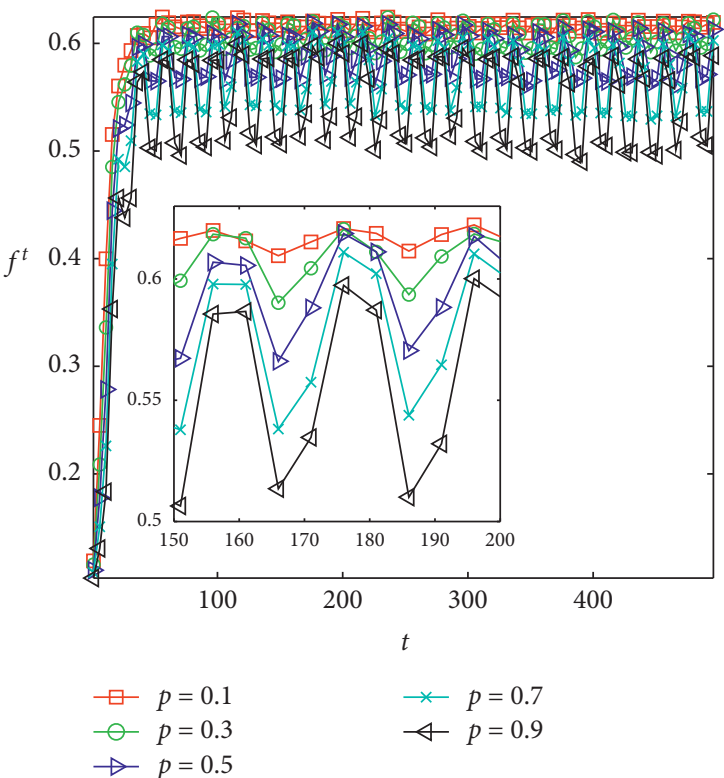

(a)

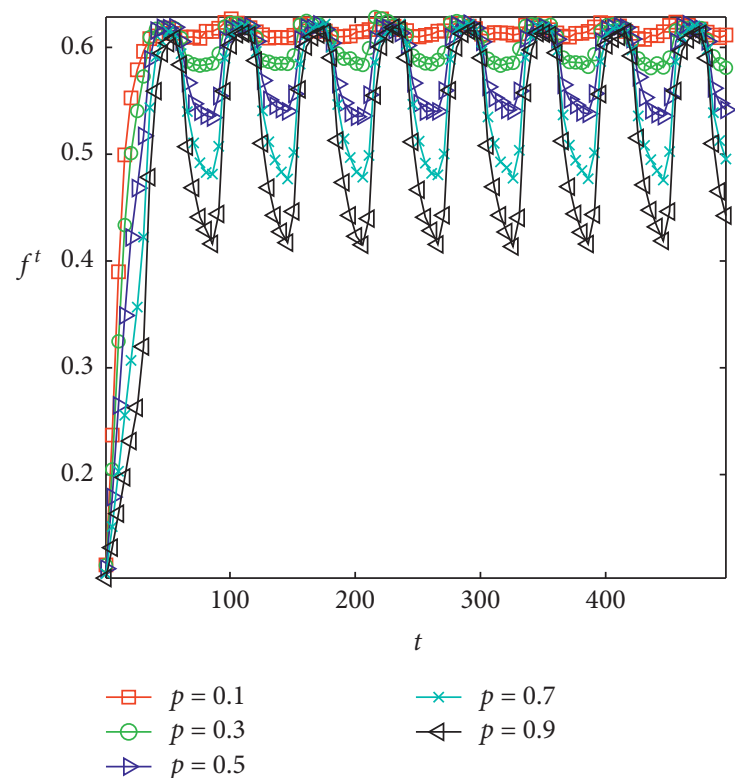

(b)

Figure 6: The evolution of the infected density $f^{t}$ with time under different value of $p$. We find the fluctuation range of the infected density increase with $p$ and $t_{s}$. The parameters are set as follows: $\alpha=0.5, \beta=0.1, \lambda_{0}=0.001$. (a) The half period $t_{s}=10$; (b) the half period $t_{s}=30$.

values of $p$ and $t_{s}$ change, the maximum infected density is almost the same. Therefore, under different individuals' mobility modes and travelling period, the peak of infected density generally does not change.

Figure 7 illustrates the change of the value of $f^{t}$ over time under different regional heterogeneous infection coefficient $\alpha$. By comparing and analyzing Figures 7(a) and 7(b), it can be found that with the increase of the regional heterogeneous infection coefficient $\alpha$, the fluctuation range of the infected density $f^{t}$ becomes larger and larger. In addition, when the infection rate is high $\left(\lambda_{0}=0.002\right)$, it is interesting to find that the fluctuation period of the infected density $f^{t}$ is almost the same under different $\alpha$. 


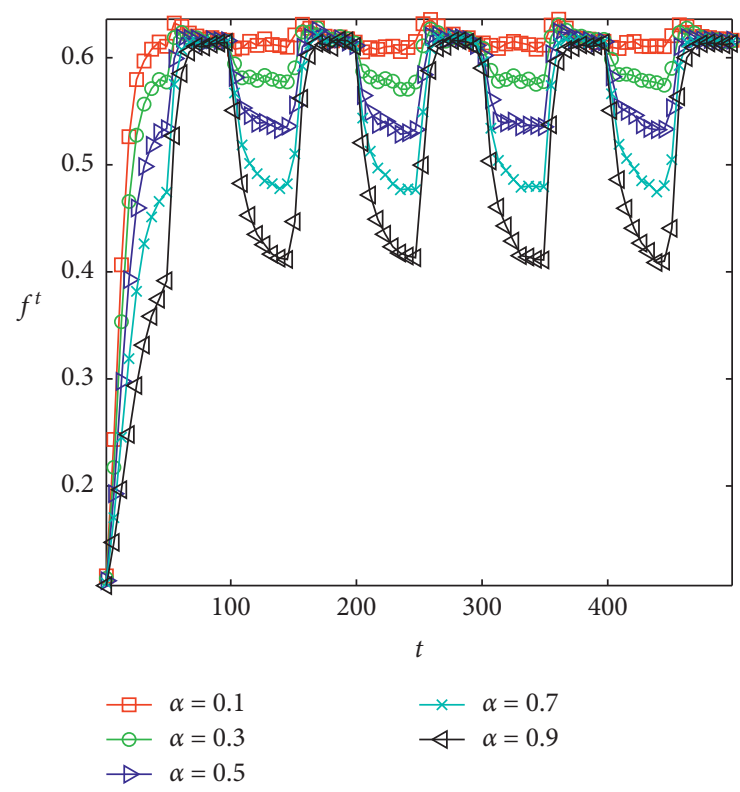

(a)

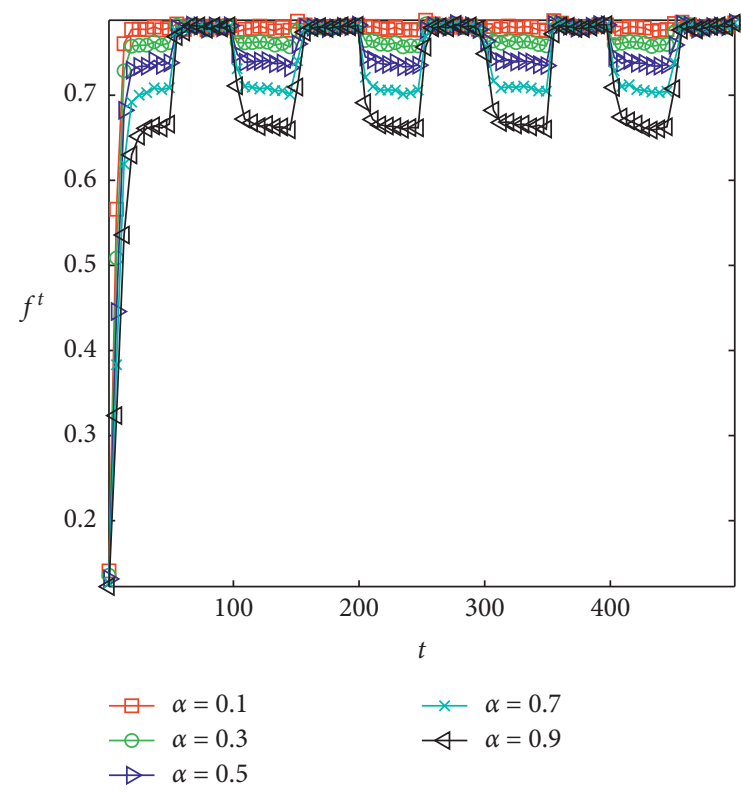

(b)

Figure 7: The infected density $f^{t}$ evolves with time under different regional heterogeneous infection coefficient $\alpha$. It is interesting to find that the fluctuation period of the infected density $f^{t}$ is almost the same under different $\alpha$. The parameters are set to be $t_{s}=50, p=0.5$, $\beta=0.1$. (a) The infection rate $\alpha_{0}=0.001$; (b) the infection rate $\lambda_{0}=0.002$.

\section{Conclusion}

The convenience of transportation brings the diversity of individual travel; that means we may not describe the individuals' mobility just by using a single layer network. However, the multilayer system and metapopulation model are the very effective tools to model it. Some researchers use airport traffic data, train traffic data, or other public transport data to simulate the spread of infectious diseases in the metapopulation network. However, some data are still unavailable. In particular, if the data of infected people cannot be obtained, it will lead to inaccurate results, so the validity and accuracy of these data need to be further improved. At present, it is more difficult to obtain real and accurate data of different travel modes. In this paper, we propose a multilayer metapopulation network model to study the epidemic spreading process. In addition, we consider the periodicity of individual commuting, exploring the epidemic spreading dynamic behavior in the periodic metapopulation network. The epidemic spreading threshold is theoretically calculated based on the Perron-Frobenius theorem. Further, we perform a large number of numerical simulations, and the simulation results are consistent with the theoretical deduction. The results show that if each individual arrives in an unbiased regions, the diffusion of infectious diseases can be effectively suppressed. Moreover, with the increase of mobility rate or regional heterogeneous infection coefficient, the fluctuation range of infected density becomes larger and larger, while the fluctuation period is almost unchanged. However, the change of individual travelling period leads to the change of fluctuation period of infected density. At present, the researches for the epidemic spreading in the metapopulation network mostly consider the single-layer network or the individual's behavior as nonperiodic movement, and our study attempts to provides a theoretical and analytical method for the study of epidemic spreading in the multilayer metapopulation network with individual periodic movement.

\section{Data Availability}

No data were used.

\section{Conflicts of Interest}

The authors declare that they have no conflicts of interest.

\section{Acknowledgments}

This research was supported by the National Nature Science Foundation of China (nos. 61803184 and 71974080); National Nature Science Foundation of Jiangsu Province (nos. BK20180851 and BK20190832); Ministry of Education of Humanities and Social Science Project (No. 20YJC630059); China Postdoctoral Science Foundation (nos. 2018M640326 and 2019M660832); Jiangsu Postdoctoral Research Funding Project (No. 2019K172); Social Science Fund of Jiangsu Province (No. 18TQD002); Major Projects of the National Social Science Fund (No. 18VSJ038); Natural Science Research Projects of Jiangsu Higher Education Institutions (No. 19KJB120001). 


\section{References}

[1] D. Bernoulli, "Essai d-une nouvelle analyse de la mortalite cause par la petite verole et des avamtages de l' inoculation pour la prevenir," Académie Royale des Sciences, Paris, France, Mémoires de Mathématique et de Physique, 1760.

[2] D. J. Watts and S. H. Strogatz, "Collective dynamics of "smallworld” networks," Nature, vol. 393, no. 6684, pp. 440-442, 1998.

[3] A.-L. Barabási and R. Albert, "Emergence of scaling in random networks," Science, vol. 286, no. 5439, pp. 509-512, 1999.

[4] M. Mancastroppa, A. Vezzani, M. A. Munoz, and R. Burioni, "Burstiness in activity-driven networks and the epidemic threshold," Journal of Statistical Mechanics-Theory and Experiment, vol. 2019, no. 5, Article ID 053502, 2019.

[5] Y. Z. Zhou, J. Zhou, G. R. Chen, and H. Eugene Stanley, "Effective degree theory for epidemic dynamics on multiplex networks," New Journal of Physics, vol. 21, no. 3, Article ID 035002, 2019.

[6] Y. Z. Zhou, C. Teng, and J. Zhou, "Effective degree theory on multiplex networks for concurrent three-state spreading dynamics," IEEE Access, vol. 7, pp. 70486-70494, 2019.

[7] H. W. Lee, N. Malik, F. Shi, and P. J. Mucha, "Social clustering in epidemic spread on coevolving networks," Physical Review E, vol. 99, no. 6, Article ID 062301, 2019.

[8] Z. X. Wang, M. Tang, S. M. Cai, Y. Liu, J. Zhou, and D. D. Han, "Self-awareness control effect of cooperative epidemics on complex networks," Chaos: An Interdisciplinary Journal of Nonlinear Science, vol. 29, no. 5, Article ID 053123, 2019.

[9] P. Zhu, Q. Zhi, Y. Guo, and Z. Wang, "Analysis of epidemic spreading process in adaptive networks," IEEE Transactions on Circuits and Systems II: Express Briefs, vol. 66, no. 7, pp. 1252-1256, 2019.

[10] L. K. Andrew, K. Lawrence, Y. Kamran, and A. V. G. Robert, "Stochastic epidemic metapopulation models on networks: SIS dynamics and control strategies," Journal of Theoretical Biology, vol. 449, pp. 35-52, 2018.

[11] S. S. Feng and Z. Jin, "Moment closure of infectious diseases model on heterogeneous metapopulation network," Advances in Difference Equations, vol. 2018, no. 1, p. 39, 2018.

[12] G. Tanaka, C. Urabe, and K. Aihara, "Random and targeted interventions for epidemic control in metapopulation models," Scientific Reports, vol. 4, no. 1, p. 5522, 2014.

[13] N. Takashi, I. Genki, and K. Tainaka, "Epidemics of random walkers in metapopulation model for complete, cycle and star graphs," Journal of Theoretical Biology, vol. 450, pp. 66-75, 2018.

[14] L. Anurag, P. E. Alfonso, A. M. Li, and J. Gore, "Asymmetric migration decreases stability but increases resilience in a heterogeneous metapopulation," Nature Communications, vol. 9, no. 1, p. 2969, 2018.

[15] D. J. Watts, R. Muhamad, D. C. Medina, and P. S. Dodds, "Multiscale, resurgent epidemics in a hierarchical metapopulation model," Proceedings of the National Academy of Sciences, vol. 102, no. 32, pp. 11157-11162, 2005.

[16] A. Anzo-Hernández, B. Bonilla-Capilla, J. Velázquez-Castro, M. Soto-Bajo, and A. Fraguela-Collar, "The risk matrix of vector-borne diseases in metapopulation networks and its relation with local and global $R_{0}$." Communications in Nonlinear Science and Numerical Simulation, vol. 68, pp. 1-14, 2019.

[17] I. Hanski and M. E. Gilpin, Metapopulation Biology: Ecology, Genetics, and Evolution, Acamemic Press, San Diego, CA, USA, 1997.
[18] A. Iggidr, G. Sallet, and B. Tsanou, "Global stability analysis of a metapopulation SIS epidemic model," Mathematical Population Studies, vol. 19, no. 3, pp. 115-129, 2012.

[19] Y. W. Gong and S. Michael, "Epidemic spreading on metapopulation networks including migration and demographics," Chaos: An Interdisciplinary Journal of Nonlinear Science, vol. 28, no. 8, Article ID 083102, 2018.

[20] T. Nagatani, G. Ichinose, and K.-i. Tainaka, "Epidemic spreading of random walkers in metapopulation model on an alternating graph," Physica A: Statistical Mechanics and Its Applications, vol. 520, pp. 350-360, 2019.

[21] N. Takashi, I. Genki, and K. Tainaka, "Metapopulation model for rock-paper-scissors game: mutation affects paradoxical impacts," Journal of Theoretical Biology, vol. 450, pp. 22-29, 2018.

[22] B. Wang, Y. Han, and G. Tanaka, "Interplay between epidemic spread and information propagation on metapopulation networks," Journal of Theoretical Biology, vol. 420, pp. 18-25, 2017.

[23] Y.-W. Gong, Y.-R. Song, and G.-P. Jiang, "Time-varying human mobility patterns with metapopulation epidemic dynamics," Physica A: Statistical Mechanics and Its Applications, vol. 392, no. 19, pp. 4242-4251, 2013.

[24] S. J. Ni and W. G. Weng, "Impact of travel patterns on epidemic dynamics in heterogeneous spatial metapopulation networks," Physical Review E, vol. 79, no. 1, 2009.

[25] B. Wang, L. Cao, H. Suzuki, and K. Aihara, "Safety-information-driven human mobility patterns with metapopulation epidemic dynamics," Scientific Reports, vol. 2, no. 1, p. 887, 2012.

[26] L. Halvor, L. Ludvig, and S. Ingve, "Effects of city-size heterogeneity on epidemic spreading in a metapopulation: a reaction-diffusion approach," Journal of Statistical Physics, vol. 151, no. 1-2, pp. 367-382, 2013.

[27] M. Naoki, "Effects of diffusion rates on epidemic spreads in metapopulation networks," New Journal of Physics, vol. 12, no. 9, Article ID 093009, 2010.

[28] B. Frank, B. Tom, H. Thomas et al., "Seven challenges for metapopulation models of epidemics including households models," Epidemics, vol. 10, pp. 63-67, 2015.

[29] A. Vespignani, "Reaction-diffusion processes and epidemic metapopulation models in complex networks," The European Physical Journal B, vol. 64, no. 3-4, pp. 349-353, 2008.

[30] J. B. Wang and X. Li, "Uncovering spatial invasion on metapopulation networks with SIR epidemics," IEEE Transactions on Network Science and Engineering, vol. 6, no. 4, pp. $788-800,2018$

[31] J. B. Wang, L. Wang, and X. Li, "Identifying spatial invasion of pandemics on metapopulation networks via anatomizing arrival history," IEEE Transactions on Cybernetics, vol. 46, no. 12, pp. 2782-2795, 2016.

[32] K. C. Chang, K. Pearson, and T. Zhang, "Perron-frobenius theorem for nonnegative tensors," Communications in Mathematical Sciences, vol. 6, no. 2, pp. 507-520, 2008. 